\title{
Next WFUMB Congress
}

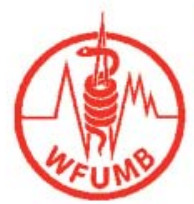

The 17th World Federation for Ultrasound in Medicine and Biology Congress

hosted by the Australasian Society for Ultrasound in Medicine

((ASUM2019 2 -9 9 September 2019

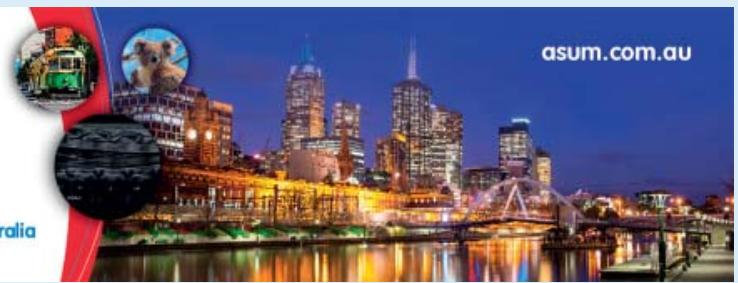

